\title{
Lateralization of tonal signals in noise
}

\author{
THOMAS F. ELLIOTT and W. A. WILBANKS \\ University of Georgia, Athens, Georgia 30602
}

\begin{abstract}
When listeners attempt to localize a tone in noise, the "signal" for lateralization is the change in interaural phase between tone plus noise and the interaural phase of the noise alone. Data are presented showing how lateralization performance varies as a function of the interaural correlation of the noise, signal-to-noise ratio, and tone frequency. The relationship between the results of this study and some phenomena of binaural signal detection is discussed.
\end{abstract}

It was just over 30 years ago that the now-classic research of Hirsh (1948) and Licklider (1948) showed that the detectability of monaural and binaural signals in noise can be improved by manipulation of those variables traditionally associated with the localization of sound sources, namely, interaural time or phase differences. The interaural phase effects found by Hirsh were quite dramatic, but they have been repeatedly verified by subsequent research. When a signal and a noise are presented to both ears and one or the other is reversed in phase, the signal can be detected at a level on the order of $15 \mathrm{~dB}$ lower than when both are in phase (e.g., Blodgett, Jeffress, \& Taylor, 1958; Jeffress, Blodgett, \& Deatherage, 1952; Jeffress, Blodgett, Sandel, $\&$ Wood, 1956). Also, the detection of a tonal signal at one ear, partially masked by noise at that ear, can be improved by as much as $10 \mathrm{~dB}$ when identical noise is added at the other ear (e.g., Blodgett, Jeffress, \& Whitworth, 1962; Mulligan \& Wilbanks, 1965; Weston \& Miller, 1965; Wilbanks \& Whitmore, 1968). In both cases, the signal to which the observer responds is too weak to be detected by monaural means.

In addition to the effects of reversing the phase of the signal or the noise, the effects of varying the magnitude of the interaural correlation for the noise on the masking of speech were investigated by Licklider (1948). The in-phase and the 180-deg out-of-phase maskers are, as Licklider pointed out, +1.00 and -1.00 productmoment correlations, respectively. Licklider found that the advantages obtained with in-phase and 180-deg out-of-phase signals became less and less as the interaural correlation for the noise was reduced by adding uncorrelated noise in the channels to the ears. Langford and Jeffress (1964) and Robinson and Jeffress (1963) have shown that the magnitude of the interaural correlation for the masking noise is a dominant variable affecting binaural unmasking when a tonal signal is presented to both ears (i.e., the reduction in masking that occurs when the signal is detected by binaural means). This

Some of the results of this study were presented at the 85 th meeting of the Acoustical Society of America. Requests for reprints should be sent to W. A. Wilbanks, Department of Psychology, University of Georgia, Athens, Georgia 30602. increase in signal detectability has been termed the binaural masking-level difference, or MLD. Wilbanks and Whitmore (1968) extended this work on binaural unmasking and noise correlation to a wide range of frequencies in which the tonal signal is presented monaurally rather than binaurally. These studies have clearly shown that as the correlation of the masking noise is reduced from unity, the size of the MLD is reduced.

The present work is based upon the assumption that both the localization of signals in noise and the detection of signals in noise depend upon the detection by the listener of the change in the interaural phase or time difference for the noise when the signal is added. In other words, the "signal" for binaural detection and localization is the same: interaural phase or time difference. With this in mind, we were interested in seeing how the ability of listeners to localize tones in noise would be modified by manipulating variables involved in binaural unmasking. Specifically, if a tonal signal is presented in noise, what is the effect of varying the interaural correlation of the noise on the detection of the signal for localization, the change in the interaural phase or time difference that results when the signal is added to the noise?

\section{METHOD}

A $250-\mathrm{Hz}$ tone was binaurally presented to the listener at a level of $74 \mathrm{~dB}$ re .0002 microbar. The interaural phase of the tone was varied using a Grason-Stadler phase shifter. The interaural phase value for a given condition was determined by measuring the corresponding interaural time difference with an Atec electronic counter. The duration of the tone $(150 \mathrm{msec})$ was controlled by an electronic clock that triggered a GrasonStadler switch, gating the tone with a 10 -msec on-off time. The level of the signal was measured across the subjects' earphones (TDH-49) with a true-rms voltmeter and a wave analyzer.

Each experimental session consisted of 100 two-alternative forced-choice trials. The sequence of events within each trial was: (1) warning interval, $100 \mathrm{msec}$; (2) pause, $800 \mathrm{msec}$; (3) first observation interval, $150 \mathrm{msec}$; (4) pause, $800 \mathrm{msec}$; (5) second observation interval, $150 \mathrm{msec}$; and (6) response period, $2 \mathrm{sec}$. The occurrence of the warning and observation intervals was marked for the observer by lights. The leading channel of the output of the phase shifter was electronically commutated so that it could be presented to either the left or the right ear. Two types of trials occurred during each 100-trial session. A Type 1 trial consisted of (1) a signal leading in phase at 
the left ear during the first observation interval and (2) a signal leading in phase at the right ear during the second observation interval. A Type 2 trial consisted of the reverse sequence of events. After each trial, the observer's task was to state which type of trial had occurred. The a priori probability of each type was .50 .

The signals were presented in a background of wide-band noise, cut off at the lower end by response of the earphones and at the upper end by a $3,000-\mathrm{Hz}$ low-pass passive filter. The noise was presented at two overall levels, $+64 \mathrm{~dB}$ and $+84 \mathrm{~dB}$ SPL re .0002 microbar. Two noise generators were used to obtain noise with positive interaural correlations of +1.00 , $+.87,+.50,+.22$, and 0 . The perfect positive correlation (NO) was achieved by using a single noise source for both ears. The zero correlation (NU) was achieved by using independent noise generators, one for each ear. Interaural correlations between unity and zero were obtained by mixing the outputs of the two noise sources in the channel to one ear, the noise at the other ear being supplied by only one of the generators. In this case, the interaural correlation is given by: $\mathrm{r}^{2}=\mathrm{e}^{2} /\left(\mathrm{e}_{\mathrm{c}}{ }^{2}+\mathrm{e}_{\mathrm{u}}{ }^{2}\right)$, where $e_{c}$ is the rms voltage common to both ears and $e_{u}$ is the rms voltage of the noise unique to one ear. The values of $e_{c}{ }^{2}$ and $\left(\mathrm{e}_{\mathrm{c}}{ }^{2}+\mathrm{e}_{\mathrm{u}}{ }^{2}\right)$ for any given correlation were equal. This way of varying interaural correlation has been thoroughly discussed by Jeffress and Robinson (1962) and Licklider and Dzendolet (1948).

Two observers were used. Since both had had considerable experience with detection and lateralization tasks, no special instructions were required, and no system of trial-by-trial feedback was used. The observers were trained sufficiently that no correction for response bias was required. All listening was done in standard IAC booths. For each experimental condition, a minimum of three values of interaural phase for the $250-\mathrm{Hz}$ tone were used, and at least 500 forced-choice trials were given for each value of interaural phase for the tone. From these data, psychometric functions were obtained relating percentage correct choices to interaural phase value. From these psychometric functions, the main dependent variable was determined: the interaural phase for the tone for $85 \%$ correct lateralization choices.

Data were also obtained with a $1,000-\mathrm{Hz}$ tone on one observer. The same conditions and procedure as described above were used.

\section{RESULTS AND DISCUSSION}

The results are given in Figure 1 and in Table 1.

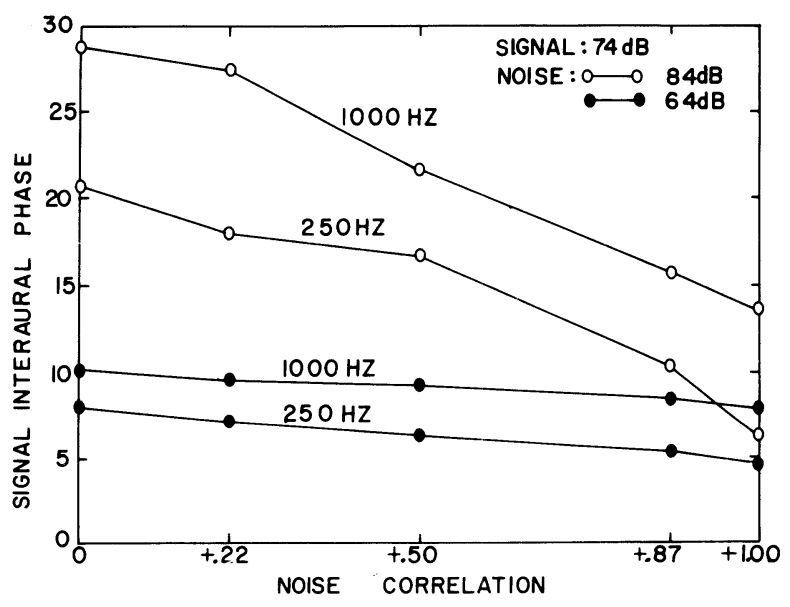

Figure 1. Average interaural phase-difference thresholds of $85 \%$ correct lateralization judgments.
Table 1

Average Interaural Phase Differences in Degrees for $85 \%$ Correct Judgments for $250-\mathrm{Hz}$ Tone

\begin{tabular}{ccc}
\hline $\begin{array}{c}\text { Noise } \\
\text { Correlation }\end{array}$ & $\begin{array}{c}64-\mathrm{dB} \\
\text { Noise }\end{array}$ & $\begin{array}{c}84-\mathrm{dB} \\
\text { Noise }\end{array}$ \\
\hline+1.00 & 5.1 & 6.8 \\
+.87 & 6.2 & 9.6 \\
+.50 & 6.4 & 16.6 \\
+.22 & 7.4 & 18.0 \\
.00 & 9.5 & 20.7 \\
\hline
\end{tabular}

Figure 1 shows the mean performance of the two subjects with the $250-\mathrm{Hz}$ tone and for the one subject with the $1,000-\mathrm{Hz}$ tone. Here, the ordinate is interaural phase in degrees for $85 \%$ correct choices, the abscissa is interaural noise correlation, and the parameter is signal frequency. The data for $250-\mathrm{Hz}$ are presented in Table 1. With an 84-dB noise, the interaural phase difference for $85 \%$ correct choices for the $250-\mathrm{Hz}$ tone increases from about $7 \mathrm{deg}$ to $21 \mathrm{deg}$ as the noise correlation is reduced from unity to zero. Although the same trend is evident in the low-noise condition $(64 \mathrm{~dB})$, the effect of reducing noise correlation is considerably less. In this condition, the interaural phase difference for $85 \%$ correct choices increases from about $5 \mathrm{deg}$ to $10 \mathrm{deg}$ as the noise correlation is reduced from unity to zero. Figure 1 indicates that an increase in noise correlation from +.50 to +.87 in the highnoise condition with the $250-\mathrm{Hz}$ tone requires a greater change in signal interaural phase difference for $85 \%$ correct choices than does the entire range of noise correlation employed with the $64-\mathrm{dB}$ noise. The same general results obtain with the $1,000-\mathrm{Hz}$ signal, although lateralization performance is poorer under all conditions.

The results clearly show that when a listener attempts to localize a tonal signal in a background of noise, a dominant variable determining the detectability of the signal for localization, interaural phase or time difference, is the interaural correlation for the noise. The effects of reducing the interaural correlation for noise are the same for detection and lateralization. The results also show that signal-to-noise ratio is a major variable affecting the localization of tonal signals in noise. This finding is in agreement with the work of Houtgast and Plomp (1968). In their study, both signal and noise were octave bands of noise with center frequencies of $500 \mathrm{~Hz}$. A constant time delay of 400 microsec was introduced into the noise channel to shift the lateral image of the masking noise away from the image of the signal. A variable time delay was introduced into the signal channel. This delayed signal could be presented to either ear by means of an automatic switch. The task required of their observers was to indicate whether the signal in the first observation interval was to the left or right of the signal in the second interval. They found that lateralization performance was degraded as the signalto-noise ratio was reduced.

The vector model of binaural detection proposed by 
Jeffress provides a convenient means of examining the signal and noise interactions as to their effects on lateralization performance (Jeffress, 1972; Jeffress, Blodgett, Sandel, \& Wood, 1956). The model begins with an examination of the statistics of the noise. The band of noise that masks the tone can be described as a sinusoid that varies slowly in phase and in amplitude (monaural critical band) and to which a signal of constant amplitude is added in random phase. The addition of the signal yields a signal-plus-noise resultant that will, in general, differ in phase from the original noise, sometimes advancing and sometimes retarding the noise in phase. If an out-of-phase signal is presented binaurally in a background of correlated noise, there will be an interaural phase difference between the signal plus noise and the noise alone. The size of the interaural phase shift will be determined by, among other things, the level of the signal relative to the level of the noise for any given phase relation at addition (i.e., signal-to-noise ratio), the interaural phase difference of the signal, and the correlation of the noise. Jeffress' model proposes that the basis for improved detection under conditions in which MLDs are obtained is the change in the correlation between the stimuli at the two ears when the signal is added to the noise. Even when a tone is completely detectable, as in the present study, the signal and noise must interact in a similar fashion. The lateral position of a low-frequency tone can be expected to depend on the phase relationships of the signal-plus-noise resultants at the two ears when the signal is added, rather than on the phase difference of the tone alone. If an observer's responses on a lateralization task are scored on the basis of tonephase relationships alone, varying noise correlation or signal-to-noise ratio can be expected to affect performance. A reduction in either signal-to-noise ratio or noise correlation would result in decrements in lateralization performance. A listener might respond correctly as to the phase relationships of the signal-plus-noise resultants at the two ears and still be scored as incorrect on the basis of tone-phase relationships. In general, the higher the signal-to-noise ratio and the larger the noise correlation, the more lateralization performance will depend upon the interaural phase of the tonal signal. Figure 2 presents, for illustrative purposes only, Jeffress' (Note 1) vector representation of the case in which a 180-deg out-of-phase signal is added to a noise whose

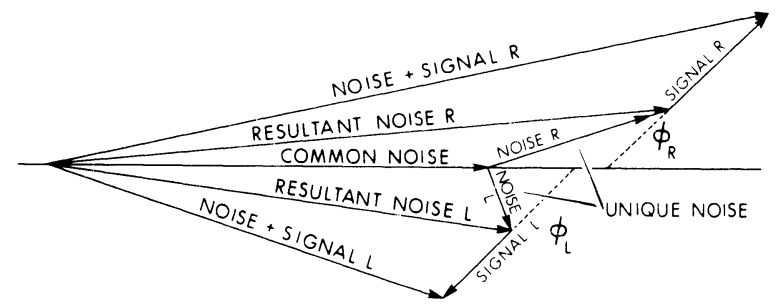

Figure 2. Vector diagram illustrating the addition of an outof-phase tone to noise whose correlation has been reduced by adding independent noise at each ear. correlation has been reduced by adding independent noise, as in the present study. Here, there is a common vector representing the common, correlated part of the noise. To this, for one ear, has been added another noise vector, representing noise from a second generator. The noise for that ear is the resultant of the two noises. For the other ear, the noise is the vector sum of the common part and a new, uncorrelated part. This yields a second resultant, which is the noise for the second ear. The amount of difference in phase and in amplitude between the noise vectors for the two ears depends upon how much the correlation has been reduced from unity. When an out-of-phase signal is added to the ears, this yields signal-plus-noise resultant vectors for each ear. The basis for both detection and localization is the change in interaural phase between noise alone and signal plus noise. The magnitude of this change depends upon the signal-to-noise ratio, the noise correlation, and the interaural phase relation of the tonal signal.

To summarize, if a tonal signal is presented in a background of noise, the listener must determine the lateral position of the signal-plus-noise resultant. With a tone of fixed intensity, the important variables are (1) interaural phase of the tone, (2) noise level, (3) noise correlation. If a very low level of correlated noise is present, the interaural phase of the signal-plus-noise resultant is determined primarily by the interaural phase of the tone. As the correlation of the noise is reduced from unity, the interaural phase of the tone must be increased in order to maintain a given level of lateralization performance, say $85 \%$ correct. If a high level of correlated noise is present, the interaural phase of the signal must be significantly increased to maintain a constant level of performance. As the correlation of the noise is reduced, again, the interaural phase of the signal must be increased. Under conditions presumably minimizing the effects of noise, Zwislocki and Feldman (1956) found that interaural phase differences on the order of 2 deg can be detected as a shift in the location of the sound image. It would appear that our low-level correlated noise only slightly masked the signal for lateralization.

When listeners attempt to localize a tone in noise, the "signal" for localization is the change in the interaural phase between the signal-plus-noise resultants at the two ears and the interaural phase of the noise alone. This "signal" can be masked, resulting in a decrement in lateralization performance, by increasing the level of the noise and, more dramatically, by reducing the interaural correlation of the noise. The phenomena of binaural unmasking and localization involve the same stimulus parameters.

\section{REFERENCE NOTE}

1. Jeffress, L. A. Masking and binaural phenomena (Report No. 245). Austin, Tex: University of Texas Defense Research Laboratory, 1965. 


\section{REFERENCES}

Blodgett, H. C., Jeffress, L. A., \& Taylor, R. W. Relation of masked thresholds to signal duration of various interaural phase-combinations. American Journal of Psychology, 1958, 71, 283-290.

Blodgett, H. C., Jeffress, L. A., \& Whitworth, R. H. Effect of noise at one ear on the masked threshold for tone at the other. Journal of the Acoustical Society of America, 1962, 34, 979-981.

Hirsh, I. J. The influence of interaural phase on interaural summation and inhibition. Journal of the Acoustical Society of America, 1948, 20, 536-544.

Houtgast, T., \& Plomp, R. Lateralization threshold of a signal in noise. Journal of the Acoustical Society of America, 1968, 44, 807-812.

Jeffress, L. A. Binaural signal detection: Vector theory. In J. V. Tobias (Ed.), Foundations of modern auditory theory (Vol. 2). New York: Academic Press, 1972.

Jeffress, L. A., Blodgett, H. C., \& Deatherage, B. H. The masking tones by white noise as a function of the interaural phases of both components. I. 500 cycles. Journal of the Acoustical Society of America, 1952, 24, 523-527.

Jeffress, L. A., Blodgett, H. C., Sandel, T. T., \& Wood, C. L. Masking of tonal signals. Journal of the Acoustical Society of America, 1956, 28, 416-426.

Jeffress, L. A., \& Robinson, D. E. Formulas for the coefficient of interaural correlation for noise. Journal of the Acoustical Society of America, 1962, 34, 1658(L).
Langford, T. L., \& Jeffress, L. A. Effect of noise crosscorrelation on binaural signal detection. Journal of the Acoustical Society of America, 1964, 36, 1455-1458.

LiCKLIDER, J. C. R. The influence of interaural phase relations upon the masking of speech by white noise. Journal of the Acoustical Society of America, 1948, 20, 150-159.

Licklider, J. C. R., \& Dzendolet, E. Oscillographic scatterplots illustrating various degrees of correlation. Science, 1948, 107, 121-124.

Mulligan, B. E., \& Wilbanks, W. A. Effect of noise at one ear upon the detection of signals at the other ear. Journal of the Acoustical Society of America, 1965, 37, 1179(A).

Robinson, D. E., \& Jefrress, L. A. Effect of varying the interaural noise correlation on the detectability of tonal signals. Journal of the Acoustical Society of America, 1963, 35, 1947. 1952.

Weston, P. B., \& Miller, J. D. Use of noise to eliminate one ear from masking experiments. Journal of the Acoustical Society of America, 1965, 37, 638-646.

Wilbanks, W. A., \& Whitmore, J. K. Detection of monaural signals as a function of interaural noise correlation and signal frequency. Journal of the Acoustical Society of America, 1968, 43, 785-797.

Zwislocki, J., \& Feldman, R. S. Just noticeable differences in dichotic phase. Journal of the Acoustical Society of America, 1956, 28, 860-864.

(Received for publication October 22, 1979.) 